\title{
Cefuroxime-Loaded Hydrogels for Prevention and Treatment of Bacterial Contamination of Open Wounds
}

\author{
Pavlo Virych $\left(\mathbb{D}\right.$, Oksana Nadtoka $\left(\mathbb{D}\right.$, Volodymyr Doroschuk $\left(\mathbb{D}\right.$, Sergey Lelyushok $\mathbb{D}^{\mathrm{D}}$,

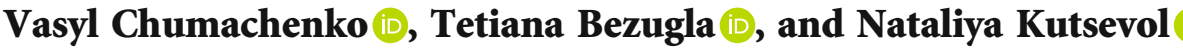

Taras Shevchenko National University of Kyiv, 64/13, Volodymyrska St, Kyiv 01601, Ukraine

Correspondence should be addressed to Pavlo Virych; sphaenodon@ukr.net

Received 12 July 2021; Revised 9 September 2021; Accepted 16 October 2021; Published 8 November 2021

Academic Editor: Deepa Kodali

Copyright (c) 2021 Pavlo Virych et al. This is an open access article distributed under the Creative Commons Attribution License, which permits unrestricted use, distribution, and reproduction in any medium, provided the original work is properly cited.

\begin{abstract}
Dextran/Sulfodextran-graft-polyacrylamide- and polyacrylamide-based hydrogels were synthesized by radical polymerization and loaded with cefuroxime to obtain antimicrobial wound dressings. Antibiotic release from the antibiotic-loaded hydrogels into an aqueous solution was studied by the HPLC-UV method. It is shown that cefuroxime-loaded Dextran/Sulfodextran-graftpolyacrylamide hydrogels release the antibiotic more slowly compared to polyacrylamide hydrogel with the same density of cross-links. Antibacterial activity of the synthesized materials was tested in vitro against wild strains of $S$. aureus, E. coli, and Klebsiella spp. The possibility of using the obtained antimicrobial hydrogels for the treatment of infected wounds was confirmed in vivo in a rat model.
\end{abstract}

\section{Introduction}

Among the many antibacterial agents, antibiotics are the most effective and widely used drugs. However, their widespread and often thoughtless use has provoked the emergence and expansion of resistant strains of bacteria. There are two main ways to overcome antibiotic resistance: the synthesis of novel antibiotics and the development of new methods of antibiotic applications. Hydrogels of different chemical nature appear to be promising carriers of antibiotics in biotechnology and medicine. Over the last few decades, various antibiotic-loaded hydrogels have been developed as antibacterial coatings and dressings for the treatment of superficial trauma, burn, or diabetic wounds [1-5]. These materials release antibiotics at the wound site, thus preventing infection and promoting the healing process [6]. Local administration of antibiotics significantly reduces the unwanted side effects that are often observed with systemic use.

Hydrogels are three-dimensional cross-linked polymer systems possessing high water swellability. The use of hydrogels as wound dressings or coverings requires some special properties such as biocompatibility, oxygen permeability, sufficient mechanical strength and flexibility, and the ability to be easily applied and removed from wounds $[7,8]$. Multiple hydrophilic groups of hydrogels ensure nonadhesion to the wound, which means painless removal of the coating. They also create a moist wound environment that is beneficial for healing [9]. Currently, various hydrogels are extensively studied as drug delivery systems, and some of them have already been used as wound dressings. The researchers highlight the advantages of hydrogels over traditional pharmaceuticals $[2,10]$.

Topical administration of antimicrobials allows formulating the hydrogels that contain high concentrations of antibiotics [9]. It is especially important for the care of infected wounds and burns. Sometimes, antibiotic-loaded hydrogels are tested against both antibiotic-susceptible and antibiotic-resistant bacteria $[11,12]$.

The hydrogels loaded with antibiotics must release the active substance in a sustained manner because successful anti-inflammatory wound treatment is directly dependent on the continuous action of antimicrobial agents. Controlled and prolonged release of the antibiotic in the affected area is the essential requirement for the prevention of biofilm formation $[6,13]$. Moreover, sustained delivery of the drug to 
the application site ensures a significant increase in time intervals needed to change the dressing on the wound. The nature of the polymer and the degree of cross-linking are believed to be the main factors that regulate the ability of hydrogels to deliver drug substances and release them at the target site. Some structure peculiarities of hydrogels can affect these processes too.

Our idea was to create polyacrylamide-based antimicrobial hydrogels for the treatment of infected wounds. These hydrogels can be loaded with the desired amount of antibiotics, and their local application for drug release provides overcoming the side effects of a systemic overdose. To vary the structure of the synthesized hydrogels, small amounts of polysaccharides were used in the syntheses as structureaffecting components. The antibiotic-loaded samples were tested in vitro against some painful bacteria and in vivo as antimicrobial dressings.

\section{Materials and Methods}

2.1. Reagents. Acrylamide (AA), three samples of Dextran with $M_{\mathrm{w}}=20000 \mathrm{~g} / \mathrm{mol}(\mathrm{D} 20), M_{\mathrm{w}}=100000 \mathrm{~g} / \mathrm{mol}$ (D100), and $M_{\mathrm{w}}=500000 \mathrm{~g} / \mathrm{mol}$ (D500), Sulfodextran with $M_{\mathrm{w}}=500000$ $\mathrm{g} / \mathrm{mol}$ (SD500), N,N ${ }^{\prime}$-methylene-bis-acrylamide (MBA), and cerium (IV) ammonium nitrate (CAN) were supplied by Sigma-Aldrich (USA). Antibiotic cefuroxime and antiseptic miramistin were purchased at a local pharmacy.

All reagents were used without further purification. Double-deionized water was used for all syntheses and procedures.

2.2. Syntheses of Hydrogels. Dextran/Sulfodextran-graftpolyacrylamide (D/SD-g-PAA) hydrogels were synthesized by free-radical polymerization in the presence of MBA as a cross-linker (Figure 1) [14, 15]. Being added into the synthesis mixture in very small amounts, Dextran/Sulfodextran affected the structure of the final product.

Dextran/Sulfodextran $\left(5 \times 10^{-4} \mathrm{mmol}\right)$ was dissolved in $24 \mathrm{~mL}$ of distilled water at $25^{\circ} \mathrm{C}$. The polysaccharide solution was stirred and bubbled with argon for $20 \mathrm{~min}$ to remove oxygen. Then, a $\mathrm{CAN} / \mathrm{HNO}_{3}$ initiator system $\left(3 \times 10^{-2} \mathrm{mmol} \mathrm{CAN} \mathrm{in} 1 \mathrm{~mL}\right.$ of $\left.0.125 \mathrm{~N} \mathrm{HNO}_{3}\right)$ was added, and the solution was stirred and bubbled with argon for another $2 \mathrm{~min}$. A monomer AA $(50 \mathrm{mmol})$ and a crosslinking agent MBA ( $0.4 \mathrm{~g}$ per $100 \mathrm{~g}$ of AA) were added to the solution. The reaction mixture was kept under argon for $24 \mathrm{~h}$. To remove the unreacted monomer, the obtained hydrogels were thoroughly washed with distilled water. Then, all samples were dried and stored at ambient temperature.

Polyacrylamide (PAA) hydrogels were synthesized under the same conditions but without the Dextran/Sulfodextran in the reaction mixture. The samples of cross-linked PAA were dried and stored as the samples of cross-linked D/SD-g-PAA.

Further in the text, the synthesized hydrogels are designated as D20-g-PAA-0.4, D100-g-PAA-0.4, D500-g-PAA0.4 , and SD500-g-PAA-0.4 according to a polysaccharide component of the obtained samples and a ratio $\mathrm{MBA}$ to AA in the syntheses.

2.3. Preparation of Antibiotic-Loaded Hydrogels. The weighed samples of the dried hydrogels were placed in an aqueous solution of cefuroxime $(166.6 \mathrm{mg} / \mathrm{mL})$ and incubated at $25^{\circ} \mathrm{C}$ for 18 hours. The amount of loaded antibiotic in the hydrogel sample was estimated by subtracting the amount of cefuroxime remaining in the solution from the initial content. All concentrations were determined by high-performance liquid chromatography with ultraviolet detection (HPLC-UV). The hydrogels swollen in this solution were used for further studies of their ability to release the antibiotic. Such samples contain an indication of cefuroxime (-Cef) in their names.

2.4. Antibiotic Release Study. The swollen samples of the antibiotic-loaded hydrogels were removed from the solution of cefuroxime, wiped with a piece of filter paper, and then placed in $25.0 \mathrm{~mL}$ of distilled water. The experiment was performed with gentle stirring at $25^{\circ} \mathrm{C}$. To determine the concentration of the antibiotic released into the solution, the aliquots of the solution were taken at regular intervals and analyzed by the HPLC-UV method. The antibiotic release experiment was performed with each antibiotic-loaded hydrogel at least three times.

2.5. Thermogravimetric Analysis (TGA). Thermogravimetric studies of the synthesized hydrogels were performed using a Netzsch TG 209 F1 Libra apparatus (Selb, Germany). The experiments were carried in a nonisothermal mode. Dry samples were heated with the heating rate of $10^{\circ} \mathrm{C} / \mathrm{min}$ and monitored for weight change in the range of $30-900^{\circ} \mathrm{C}$. The measurements were carried out under nitrogen protective flow. The weight of the samples under investigation was $10 \mathrm{mg}$. $\mathrm{Al}_{2} \mathrm{O}_{3}$ served as reference material.

2.6. In Vitro Tests for Antibacterial Activity. To test the antibacterial hydrogels for their ability to inhibit bacterial growth, wild-type strains of Staphylococcus aureus, Escherichia coli, and Klebsiella spp. selectively grown on Endo's or yolk-salt agar media were used. The sensitivity of the microorganisms was evaluated by the disc diffusion method on a Müller-Hinton agar of the following composition $(\mathrm{g} / \mathrm{L})$ : agar-17, hydrolyzed casein-17.5, hydrolyzed bovine hart-2, and water-soluble starch-1.5; $\mathrm{pH}=7.3$. The dry hydrogels were swollen $(24 \mathrm{~h})$ in an aqueous solution of cefuroxime $(0.1$ and $1 \mathrm{mg} / \mathrm{mL})$. A miramistin solution $(0.1 \mathrm{mg} / \mathrm{mL})$ was used for the control experiment. The tested samples were $5 \mathrm{~mm}$ in diameter, and their sizes were similar to those of standard disks with antibiotics. The measurement of the growth inhibition zone was carried out using a digital caliper Miol 15-240. The contact of the hydrogel samples with the microorganisms occurred within $24 \mathrm{~h}$ before recording the diameter of growth inhibition. Statistical data processing was performed using the Shapiro-Wilk test $(p>0.05)$ and the Scheffe ANOVA test $(p<0.05)$. Each in vitro experiment was repeated three times. 


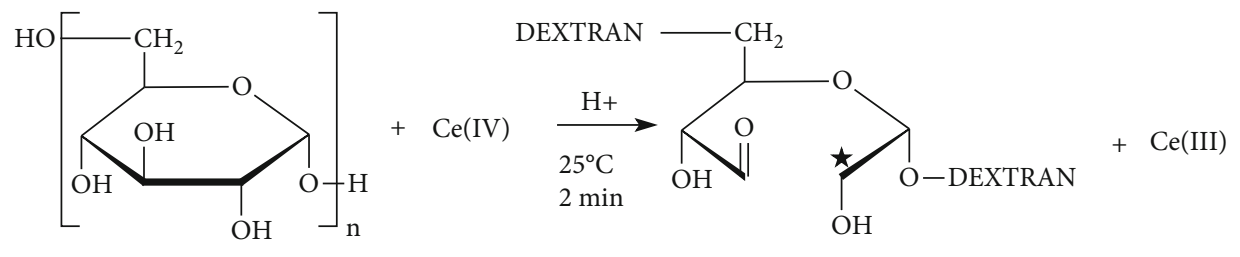

DEXTRAN

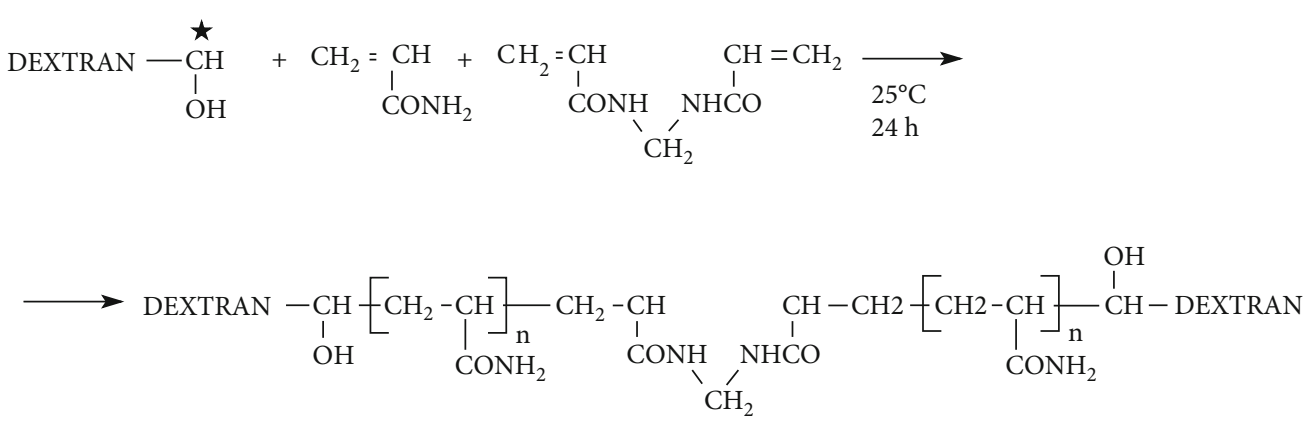

Cross-linked polymer

FIGURE 1: The proposed mechanism of the synthesis of cross-linked D-g-PAA hydrogels.

2.7. In Vivo Evaluation of Healing Properties. The effects of synthesized antibacterial hydrogels on infected wound healing were assessed by the rat wound model. The in vivo tests were performed using white outbred rats, which were kept in standard conditions of a vivarium. All manipulations with animals were carried out according to the Law of Ukraine "On the Protection of Animals from Cruelty" and the European Convention for the Protection of Vertebrate Animals Used for Research and Other Scientific Purposes.

Female rats weighing 230-260 g were selected, and general anesthesia was performed with sodium etaminal at the rate of $3.5 \mathrm{mg}$ per $1 \mathrm{~kg}$ of live weight. The area of the back between the shoulder blades of the animal was cleared of hair. A section of skin $7-8 \mathrm{~mm}$ in diameter was removed with a sharp scalpel. The resulting wound was infected with the mixture of microorganisms S. aureus, E. coli, and Klebsiella spp., which were in physiological solution in the amount of $1 \cdot 10^{8} \mathrm{CFU} / \mathrm{mL}$. The wound was covered with the synthesized hydrogels loaded with cefuroxime $(1 \mathrm{mg} / \mathrm{mL})$ or miramistin $(0.1 \mathrm{mg} / \mathrm{mL})$ and the standard fabric material (gauze dressing). The bandage was fixed with a wide Band-Aid. After $24 \mathrm{~h}$, the wound was wiped with a sterile swab, and the wound bacteria were inoculated into the nutrient medium. Yolk-saline agar was used to cultivate gram-positive bacteria, and Endo's medium was used for gram-negative bacteria.

Wound healing of the rats was assessed visually.

Skin irritation tests revealed that D/SD-g-PAA hydrogels per se did not cause any irritation, indicating its safety for topical application.

\section{Results and Discussion}

The syntheses of the cross-linked Dextran (Sulfodextran)graft-polyacrylamide hydrogels include two stages: (1) the

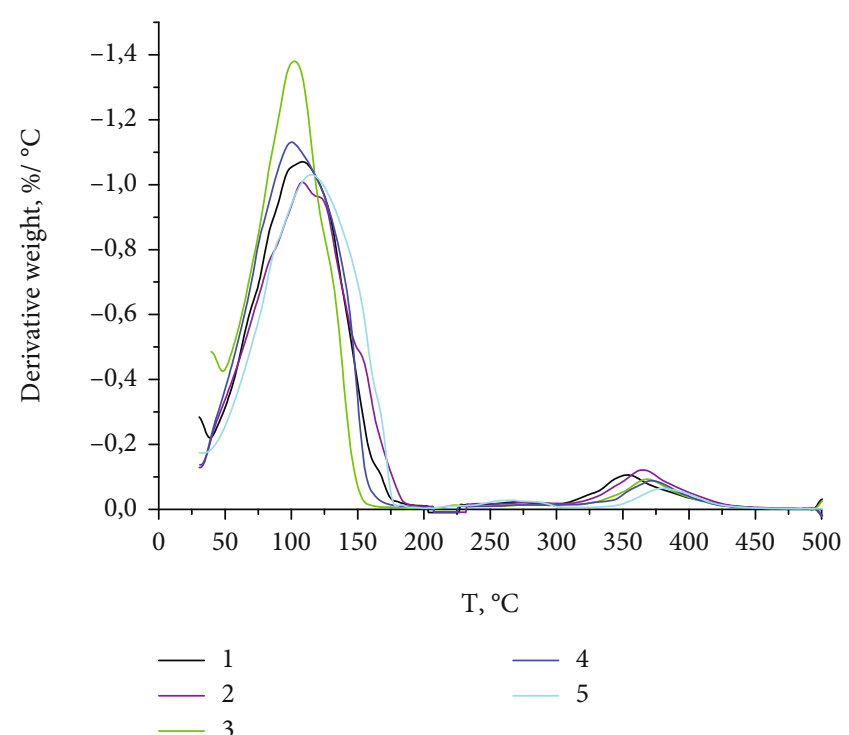

Figure 2: Derivative weight loss curve vs. temperature: (1) PAA0.4 , (2) D20-g-PAA-0.4, (3) D100-g-PAA-0.4, (4) D500-g-PAA0.4 , and (5) DS500-g-PAA-0.4 hydrogels.

preparation of polysaccharide macroradicals as a result of Dextran/Sulfodextran interaction with $\mathrm{CAN} / \mathrm{HNO}_{3}$ oxidative system $[16,17]$ and (2) the growth of PAA chains from active centers on the polysaccharide and their simultaneous cross-linking by the MBA cross-linking agent (Figure 1). Since the concentration of the cross-linking agent MBA is the same in all syntheses, it is assumed that the density of cross-links in all hydrogels is the same.

As shown earlier [18], the number of grafted polyacrylamide chains in the synthesized $\mathrm{D}(\mathrm{SD})$-g-PAA copolymers is determined by the molar ratio of the polysaccharide and 
TABLE 1: Temperature intervals of weight loss on heating, obtained by the DTA method.

\begin{tabular}{lccc}
\hline & Sample & Peak 1 & Peak 2 \\
\hline 1 & D20-g-PAA-0.4 & $35-190$ & $315-485$ \\
2 & D100-g-PAA-0.4 & $45-160$ & $320-450$ \\
3 & D500-g-PAA-0.4 & $30-180$ & $330-450$ \\
4 & DS500-g-PAA-0.4 & $30-185$ & $350-480$ \\
5 & PAA-0.4 & $35-190$ & $300-470$ \\
\hline
\end{tabular}

TABLE 2: Content of cefuroxime in the synthesized hydrogels (g of antibiotic per $\mathrm{g}$ of dried hydrogel).

\begin{tabular}{lcc}
\hline & Sample & Content $(\mathrm{g} / \mathrm{g})$ \\
\hline 1 & D20-g-PAA-0.4-Cef & 3.52 \\
2 & D100-g-PAA-0.4-Cef & 3.40 \\
3 & D500-g-PAA-0.4-Cef & 3.34 \\
4 & DS500-g-PAA-0.4-Cef & 3.31 \\
5 & PAA-0.4-Cef & 1.64 \\
\hline
\end{tabular}

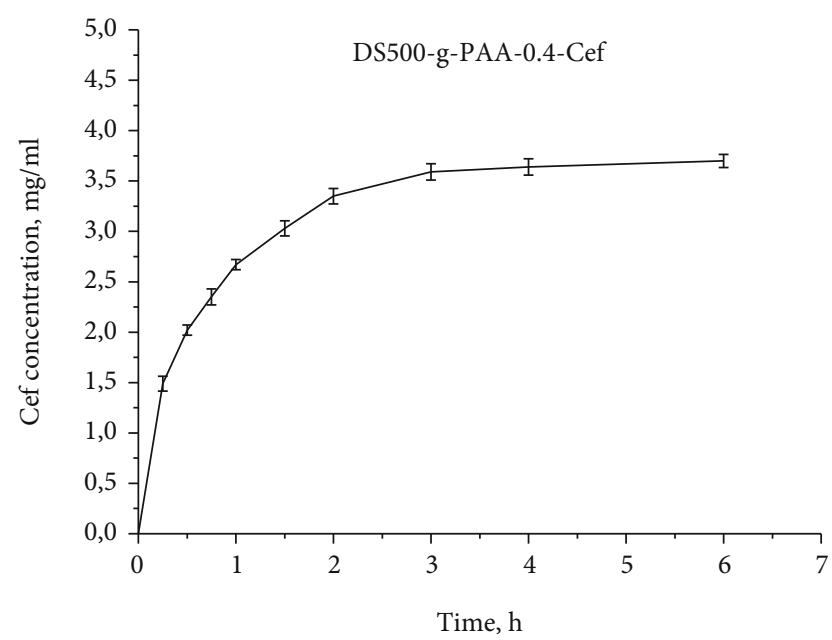

FIGURE 3: Cefuroxime concentration in water solution vs. time of contact with DS500-g-PAA-0.4-Cef hydrogel.

the CAN initiator. Formed under the action of the initiator, the active centers on the polysaccharide macromolecule trigger the growth of polyacrylamide chains. According to the molar ratio of Dextran (Sulfodextran) to CAN used in the syntheses of hydrogels described above, approximately 60 points of growth can appear on the polysaccharide macromolecule. Near the grafting point, the growing polyacrylamide chains have a conformation that essentially differs from those for PAA macromolecules in solution [16, 19]. An increase in the molecular weight of Dextran molecules, as well as the appearance of a charge on the polymer chain of Sulfodextran, has a significant effect on the conformation of the growing polyacrylamide chain in the nearest vicinity of the polysaccharide. Thus, the internal structure of the cross-linked D/SD-g-PAA hydrogels and their properties may differ from the structure and properties of crosslinked PAA with the same number of cross-links.
TABLE 3: Release of cefuroxime (in \%) from the antibiotic-loaded hydrogels into water.

\begin{tabular}{ccccc}
\hline & Sample & \multicolumn{3}{c}{ Time of contact } \\
& & $0.5 \mathrm{~h}$ & $1 \mathrm{~h}$ & $6 \mathrm{~h}$ \\
\hline 1 & PAA-0.4-Cef & $28.2 \pm 1.5$ & $41.6 \pm 3.1$ & $55.1 \pm 2.7$ \\
2 & D20-g-PAA-0.4-Cef & $24.4 \pm 2.3$ & $29.6 \pm 2.1$ & $41.9 \pm 1.6$ \\
3 & D100-g-PAA-0.4-Cef & $22.1 \pm 2.3$ & $27.0 \pm 2.2$ & $38.1 \pm 2.4$ \\
4 & D500-g-PAA-0.4-Cef & $18.6 \pm 2.7$ & $33.9 \pm 2.1$ & $39.5 \pm 2.7$ \\
5 & DS500-g-PAA-0.4-Cef & $27.9 \pm 2.5$ & $37.2 \pm 2.0$ & $50.6 \pm 2.7$ \\
\hline
\end{tabular}

TABLE 4: Antimicrobial activity of cefuroxime-loaded hydrogels swollen in $1 \mathrm{mg} / \mathrm{mL}$ antibiotic solution.

\begin{tabular}{lcccc}
\hline & \multirow{2}{*}{ Sample } & \multicolumn{3}{c}{ Diameter of the growth inhibition } \\
& & S. aureus & E. coli & Klebsiella sp. \\
\hline 1 & PAA-0.4-Cef & $27.1 \pm 1.5$ & $23.2 \pm 2.8$ & $20.1 \pm 2.4$ \\
2 & D20-g-PAA-0.4-Cef & $26.0 \pm 2.4$ & $21.1 \pm 3.0$ & $20.3 \pm 2.4$ \\
3 & D100-g-PAA-0.4-Cef & $29.8 \pm 2.1$ & $21.8 \pm 2.7$ & $20.2 \pm 2.5$ \\
4 & D500-g-PAA-0.4-Cef & $25.2 \pm 2.5$ & $22.1 \pm 2.8$ & $22.7 \pm 2.4$ \\
5 & DS500-g-PAA-0.4-Cef & $29.1 \pm 2.5$ & $21.3 \pm 2.7$ & $22.0 \pm 2.8$ \\
\hline
\end{tabular}

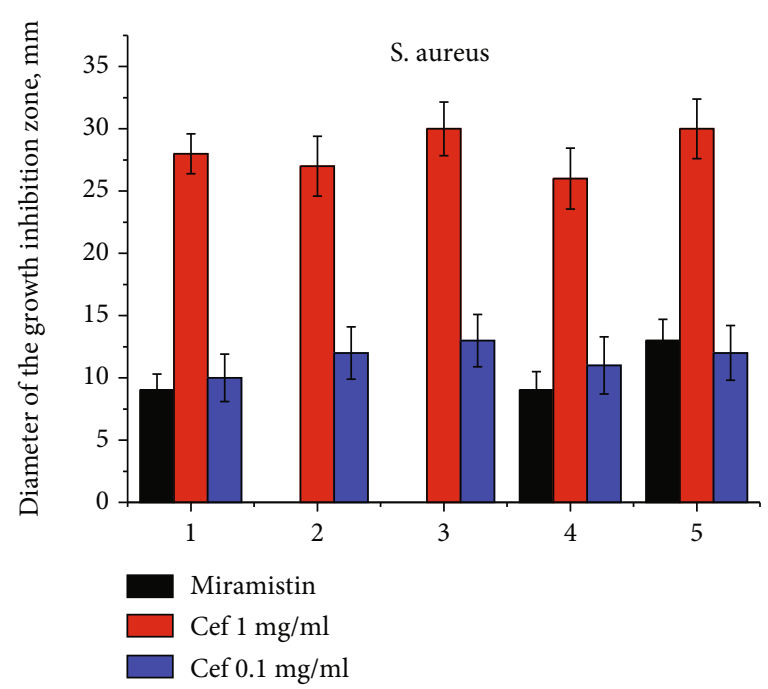

FIgURE 4: Activity of the antimicrobial hydrogels (1) PAA-0.4-Cef, (2) D20-g-PAA-0.4-Cef, (3) D100-g-PAA-0.4-Cef, (4) D500-gPAA-0.4-Cef, and (5) DS500-g-PAA-0.4-Cef against $S$. aureus (black-miramistin $0.1 \mathrm{mg} / \mathrm{mL}$; blue-cefuroxime $0.1 \mathrm{mg} / \mathrm{mL}$; red-cefuroxime $1 \mathrm{mg} / \mathrm{mL}$ ).

According to thermogravimetric data, all synthesized hydrogels are very hydrophilic and contain water even when dried. The first weight loss step is registered in the range of $40-190^{\circ} \mathrm{C}$ with a maximum of about $100^{\circ} \mathrm{C}$ indicating water desorption (Figure 2). In addition, in this interval at higher temperatures, the elimination of $\mathrm{NH}_{3}$ and $\mathrm{CO}$ can occur as a result of chemical reactions between some neighboring functional groups of the polyacrylamide chains [20]. The second distinct weight loss step is observed at $300-485^{\circ} \mathrm{C}$ with a rather 


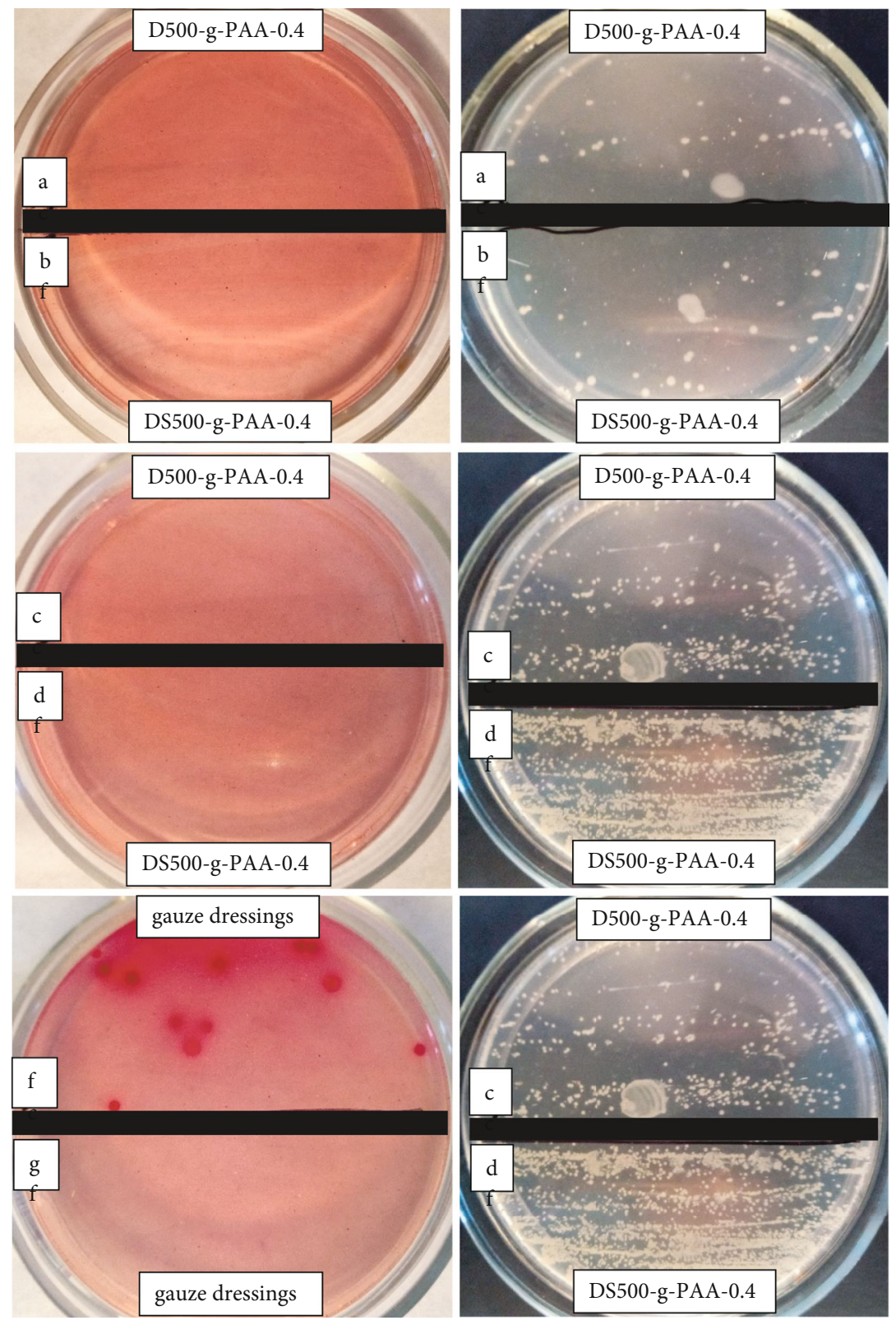

Figure 5: Cultivation of bacteria using Endo (left) and yolk-salt (right) agar media after removal of (a, b) cefuroxime-loaded hydrogels from infected wound; (c, d) miramistin-loaded hydrogels; (f, g) gauze dressings.

lower maximum on the derivative weight loss curve. It corresponds to the decomposition of the polymer matrix itself, which consists mainly of polyacrylamide fragments. The destruction of the polymer matrix of D/SD-g-PAA-0.4 samples begins at a higher temperature compared to PAA-0.4. The increase in this temperature is $15-50^{\circ} \mathrm{C}$, depending on the internal structure of the hydrogels (Table 1 ).

To prepare the antibiotic-loaded hydrogels for drug release studies, the samples of the dry hydrogels were swollen in an aqueous solution of cefuroxime for $18 \mathrm{~h}$. As shown in Table 2, the content of cefuroxime is the greatest for the hydrogels that were synthesized using Dextrans as a structure-affecting component.
The contact of the synthesized antibiotic-loaded hydrogels with water leads to the desorption of drug molecules into the solution. A typical antibiotic release profile is shown in Figure 3. For all samples, the initial burst of release is followed by desorption at a slower rate. After 3 hours, only a slight increase in the concentration of cefuroxime in the solution can be registered. The sample PAA-0.4-Cef was found to have the highest release rate; the content of cefuroxime in this hydrogel decreased by $55 \%$ after $6 \mathrm{~h}$ of contact with water (Table 3). The behavior of D-g-PAA-0.4-Cef hydrogels in this process is somewhat different. Being initially more loaded with cefuroxime, these samples release the drug into solution more slowly than PAA-0.4-Cef. 


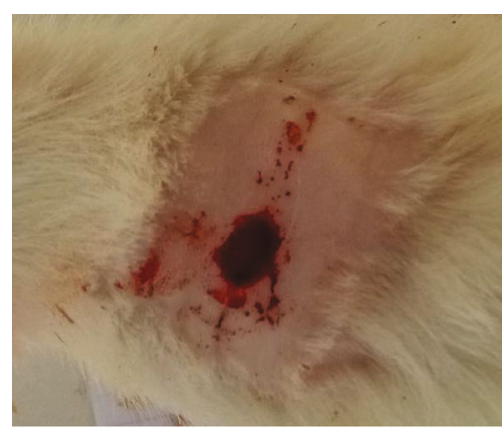

(a)

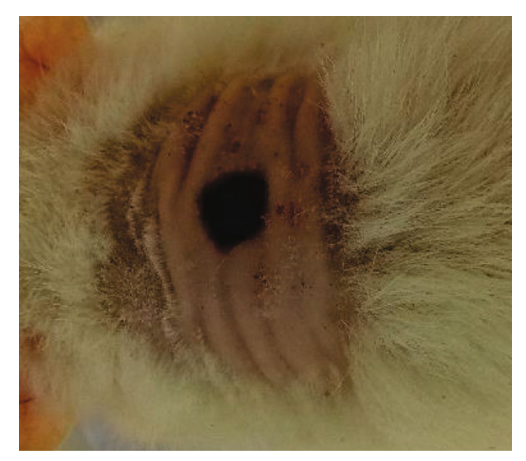

(b)

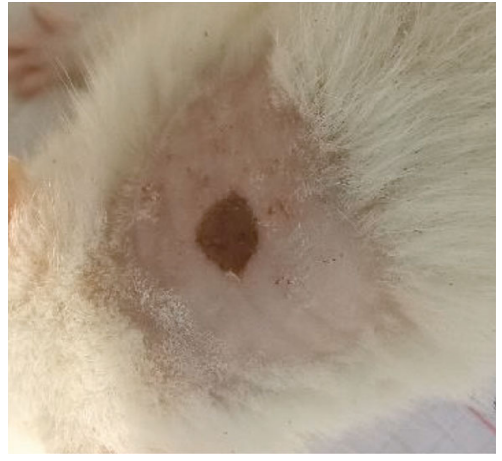

(c)

FIgURE 6: Rat wounds after removal of bandages ( $24 \mathrm{~h})$ : (a) classic gauze dressing (the wound was opened after the animal's motor activity); (b) miramistin-loaded D500-g-PAA-0.4 hydrogel; (c) cefuroxime-loaded D500-g-PAA-0.4 hydrogel.

Thus, D/DS-g-PAA-0.4-Cef hydrogels have some advantages over other hydrogels under investigation. They are assumed to be promising biomaterials for topical application as antimicrobial dressings with prolonged action.

To compare the antimicrobial activity of the antibioticloaded hydrogels with different internal structures, the dry samples were swollen in an aqueous solution of cefuroxime $(0.1$ or $1 \mathrm{mg} / \mathrm{mL})$ for $24 \mathrm{~h}$. Miramistin was used as cargo for control experiments. All synthesized cefuroxime-loaded hydrogels demonstrate high activity against test bacterial strains of S. aureus, E. coli, and Klebsiella spp. The diameter of the growth inhibition zone is $25-30 \mathrm{~mm}$ for $S$. aureus and 20-23 mm for E. coli and Klebsiella spp. when using the hydrogel samples prepared by swelling in a solution of cefuroxime with a concentration of $1 \mathrm{mg} / \mathrm{mL}$ (Table 4).

The antimicrobial activity is dose-dependent, but even at a low dose, antibiotic-loaded hydrogels demonstrate high efficacy against harmful microorganisms (Figure 4).

The in vivo study of the activity of the synthesized antibiotic-loaded hydrogels against infections was carried out on a rat wound model. Rat models are widely used to investigate various treatments for living organisms [21, 22]. To evaluate the possibility of using antibiotic-loaded D/SDg-PAA hydrogels in wound protecting and healing, two hydrogels D500-g-PAA-0.4 and SD500-g-PAA-0.4 were swollen in cefuroxime solution $(1 \mathrm{mg} / \mathrm{mL})$, and then, they were used to cover the infected wounds of rats. After 24 hours, the bandage was removed from the infected wound, and the surface material of the wound was scraped off. Inoculation of scraping microorganisms on a nutrient medium shows the complete absence of gram-negative bacteria (Figures 5(a) and 5(b), left) and very few colonies of gram-positive colonies (Figures 5(a) and 5(b), right) after $24 \mathrm{~h}$ of their growth. According to obtained data, the studied cefuroxime-loaded hydrogel samples demonstrate a higher effectiveness against bacteria compared to the miramistin-loaded hydrogels (Figures 5(c) and 5(d)) and especially to classic gauze dressings (Figures 5(f) and 5(g)).

The cefuroxime-loaded D/SD-g-PAA hydrogels demonstrated their efficacy against a mixture of pathogenic bacteria, so they can be used topically as antimicrobial dressings. The in vivo experiments show that these antimicrobial hydrogels substantially inhibit the growth of microorganisms on an infected wound surface. The appearance of the rat wounds after removing the bandage confirms the promising use of antibiotic-loaded hydrogels as biomaterials that provide good healing of superficial wounds (Figure 6).

\section{Conclusions}

D/SD-g-PAA-based hydrogels are promising materials for the development of antimicrobial dressings for the protection and treatment of superficial wounds. As shown, the structure of hydrogels can be controlled at the stage of synthesis. These hydrogels can be loaded with the desired amount of antibiotics, ensuring that the drug is delivered to the desired site without systemic overdose. The synthesized antimicrobial hydrogels can be prepared in the shape and size required for the wound. It has been shown that the release of the antibiotic is slower for antibiotic-loaded D/SD-g-PAA hydrogels compared to PAA-based hydrogel with the same number of cross-links, which is beneficial for wound healing. Testing of cefuroxime-loaded hydrogels on infected rat wounds has shown rapid clearance and healing of the infected wounds.

\section{Data Availability}

Data are available on request. To take data, please write to the corresponding author.

\section{Conflicts of Interest}

The authors declare that they have no conflicts of interest.

\section{Acknowledgments}

This publication is supported in part by the Ministry of Education and Science of Ukraine, Project (2019-2021), "Fundamental principles of creating nanohybrid functional composites synthesized in polymer matrices capable to response to external stimuli" and by the National Research Foundation of Ukraine, Project 2020.02/0022, "Plasmon hybrid nanosystems "metalpolymer-fluorophore" with enhanced optical response for 
photonics and biomedical applications" and Grant of the Ministry of Education and Science of Ukraine for perspective development of a scientific direction "Mathematical sciences and natural sciences" at Taras Shevchenko National University of Kyiv.

\section{References}

[1] S. Veiga and J. P. Schneider, "Antimicrobial hydrogels for the treatment of infection," Biopolymers, vol. 100, no. 6, pp. 637644, 2013.

[2] S. Li, S. Dong, W. Xu et al., "Antibacterial hydrogels," Advanced science (Weinheim, Baden-Wurttemberg, Germany), vol. 5, no. 5, p. 1700527, 2018.

[3] A. S. Zakaria, S. A. Afifi, and K. A. Elkhodairy, "Newly developed topical cefotaxime sodium hydrogels: antibacterial activity and in vivo evaluation," BioMed Research International, vol. 2016, 2016.

[4] H. Namazi, R. Rakhshaei, H. Hamishehkar, and H. S. Kafil, "Antibiotic loaded carboxymethylcellulose/MCM-41 nanocomposite hydrogel films as potential wound dressing," International Journal of Biological Macromolecules, vol. 85, pp. 327-334.

[5] V. Pawar, M. Dhanka, and R. Srivastava, "Cefuroxime conjugated chitosan hydrogel for treatment of wound infections," Colloids and Surfaces B: Biointerfaces, vol. 173, pp. 776-787, 2019.

[6] K. Yang, Q. Han, B. Chen et al., "Antimicrobial hydrogels: promising materials for medical application," International Journal of Nanomedicine, vol. Volume 13, pp. 2217-2263, 2018.

[7] B. Gupta, R. Agarwal, and M. S. Alam, "Hydrogels for wound healing applications," in Biomedical Hydrogels, S. Rimmer, Ed., pp. 184-227, Woodhead Publishing, 2011.

[8] S. Dhivya, V. V. Padma, and E. Santhini, "Wound dressings - a review," Biomedicine, vol. 5, no. 4, pp. 24-28, 2015.

[9] J. M. Grolman, M. Singh, D. J. Mooney, E. Eriksson, and K. Nuutila, "Antibiotic-containing agarose hydrogel for wound and burn care," Journal of Burn Care \& Research, vol. 40, no. 6, pp. 900-906, 2019.

[10] A. Francesko, P. Petkova, and T. Tzanov, "Hydrogel dressings for advanced wound management," Current Medicinal Chemistry, vol. 25, no. 41, pp. 5782-5797, 2018.

[11] P. Kaur, V. S. Gondil, and S. Chhibber, "A novel wound dressing consisting of PVA-SA hybrid hydrogel membrane for topical delivery of bacteriophages and antibiotics," International Journal of Pharmaceutics, vol. 572, p. 118779, 2019.

[12] E. Tamahkar, B. Özkahraman, A. K. Süloğlu, N. İdil, and I. Perçin, "A novel multilayer hydrogel wound dressing for antibiotic release," Journal of Drug Delivery Science and Technology, vol. 58, p. 101536, 2020.

[13] A. Shahzad, A. Khan, Z. Afzal, M. F. Umer, J. Khan, and G. M. Khan, "Formulation development and characterization of cefazolin nanoparticles-loaded cross-linked films of sodium alginate and pectin as wound dressings," International Journal of Biological Macromolecules, vol. 124, pp. 255-269, 2019.

[14] O. Nadtoka, N. Kutsevol, V. Krysa, and B. Krysa, "Hybrid polyacryamide hydrogels: synthesis, properties and prospects of application," Molecular Crystals and Liquid Crystals, vol. 672 , no. 1 , pp. 1-10, 2018.
[15] O. Nadtoka, P. Virych, T. Bezugla et al., Antibacterial Hybrid Hydrogels Loaded with Nano Silver, Applied Nanoscience, 2021.

[16] N. Kutsevol, T. Bezugla, M. Bezuglyi, and M. Rawiso, "Branched dextran-graft-polyacrylamide copolymers as perspective materials for nanotechnology," Macromolecular Symposia, vol. 317-318, no. 1, pp. 82-90, 2012.

[17] N. Kutsevol and T. Bezugla, "Influence of structural peculiarities of dextran sulphate-g-polyacrylamide on flocculation phenomen," Ecological Chemistry and Engineering S, vol. 2, no. 2, pp. 251-256, 2012.

[18] N. V. Kutsevol, T. N. Bezuglaya, and N. Y. Bezuglyi, "Features of the intramolecular structure of branched polymer systems in solution," Journal of Structural Chemistry, vol. 55, no. 3, pp. 548-559, 2014.

[19] N. Kutsevol, J. M. Guenet, N. Melnyk, D. Sarazin, and C. Rochas, "Solution properties of dextran-polyacrylamide graft copolymers," Polymer, vol. 47, no. 6, pp. 2061-2068, 2006.

[20] N. V. Kutsevol, T. B. Zheltonozhskaya, O. V. Demchenko, L. R. Kunitskaya, and V. G. Syromyatnikov, "Effect of the structure of poly(vinyl alcohol)-graft-polyacrylamide copolymers on their thermooxidative stability," Polymer Science, Series A, vol. 46, no. 5, pp. 518-525, 2004.

[21] W. A. Dorsett-Martin, "Rat models of skin wound healing: a review," Wound Repair and Regeneration, vol. 12, no. 6, pp. 591-599, 2004.

[22] L. Parnell and S. W. Volk, "The evolution of animal models in wound healing research: 1993-2017," Advances in Wound Care, vol. 8, no. 12, pp. 692-702, 2019. 